\title{
The rod as super-element of the subsystem
}

\author{
Oleg Kovalchuk ${ }^{1, *}$ \\ ${ }^{1}$ Moscow State University of Civil Engineering, 129337 Yaroslavskoe Sh.26, Moscow, Russia
}

\begin{abstract}
The article reviews some aspects of simulation of static and dynamic conditions of building bearing structures due to its necessity for durability estimation. The main method of this simulation is Finite SuperElement Method (FSEM), which is characterized by its main principle «One rod - one super-element». This element, called a super-element, may be a part of a larger multi-body system or it may be an entire mechanical system. The main algorithm of FSEM modelling focused on possible variation of bearing structure is given.
\end{abstract}

\section{Introduction}

For the simulation core supporting structure the most suitable method of super-elements (FEM) in the variant of the method of the displacements. [1,2] In this case, due to the use of the Lagrange\&D'Alembert variational principle and arbitrary functions of shape the resolving set of equations has the meaning of static or dynamic equations of equilibrium with symmetrical stiffness and mass matrices. Every finite element is described with its own stiffness matrix, load vector and mass matrix (for dynamic analysis only). The structure model is a result of the summation of lines of all elements stiffness matrices, representing internal forces projections and the summation of vector load components, representing external forces projections on global coordinates system, acting on the nodes of one element.

The feature of the integration process is that all external and internal forces, acting from the all the finite elements, containing one common node, are summed up for this node.

An integration of all nodal unknown variable (node displacements) for each node is the most rational. For rod systems it is attributed six degrees of freedom to each node, three of them are linear displacements and three other are angle of rotation relative to the global coordinate axes.

\section{The algorithm of condition estimation of rod bearing structure}

If the number of the node is known, its degree of freedom position of node displacement vector is estimated as in the following equation:

$$
I_{c c}=6(\text { Nnode }-1)+i_{c c}
$$

where $I_{c c}$ - the degree of freedom index of node displacement vector, icc - the degree of

*Corresponding author: Kovalchuk@mgsu.ru 
freedom index of node displacement vector of the single node.

The algorithm submitted is effective in the simulation of two- and three-dimensional bodies. For example, it is implemented, for example, in ANSYS.

At the same time, the use of this algorithm is complicated for modelling rod systems, where you can change the structure: extra degree of freedom appear and rearrangement of the matrix stiffness of an ensemble is required; moreover, it is required when a defect occurs in one or more rods. [4, 5] The paper [6] proposed one of the methods of formulation ratios for FE using analytical approaches.

A version of FSEM, suitable for durability of bearing system analysis problems with features given above, is shown below. The meaning of this version is equivalent to the method of joints, for example, in [7,8]. The meaning of its resolving set of equations is the same as for classic FEM or FCEM. The difference of the proposed method from the classic versions of FEM and FCEM is formulated.

Assume that Stiffness Matrix of all the FE (or SE) is known, for example, in the form of algorithms. Imagine the vector of the nodal displacements arranged in the rods:

$$
\begin{aligned}
& \mathbf{U}=\left\{\begin{array}{llll}
\mathbf{U}_{1} & \mathbf{U}_{2} & \ldots & \mathbf{U}_{N}
\end{array}\right\} \\
& \mathbf{U}_{k}=\left\{\mathbf{U}_{1 k} \quad \mathbf{U}_{2 k}\right\} k=1,2,3 \ldots N_{\text {Rods }} \\
& \mathbf{U}_{i k}=\left\{\begin{array}{llllll}
u_{x i k} & u_{y i k} & u_{z i k} & \theta_{x i k} & \theta_{y i k} & \theta_{z i k}
\end{array}\right\}, i=1,2
\end{aligned}
$$

Here $k$ is the number of terminal, $i=1$ - the beginning of the $k$-th shaft, $i=2-$ the end of the k-th terminal, $N_{\text {Rods }}-$ the number of rods in the system. The same structure, suppose for the vector of the right side. If members are Autonomous, not linked to each other, then the resolving system of equations has a block-diagonal matrix composed of stiffness matrix finite elements (SMFE) in the order of their numbering:

$$
\begin{aligned}
& \mathrm{K} \mathbf{U}=\mathbf{R} \\
& \mathrm{K}=\left[\begin{array}{cccc}
\mathrm{K}_{1} & 0 & \ldots & 0 \\
0 & \mathrm{~K}_{2} & \ldots & 0 \\
\ldots & \ldots & \ldots & \ldots \\
0 & 0 & \ldots & \mathrm{K}_{N_{\text {Rods }}}
\end{array}\right] \mathbf{R}=\left\{\begin{array}{c}
\mathbf{R}_{1} \\
\mathbf{R}_{2} \\
\ldots \\
\mathbf{R}_{N}
\end{array}\right\}
\end{aligned}
$$

the dimensions of the matrix $\mathrm{K}-12 \cdot N_{\text {Rods }} \times 12 \cdot N_{\text {Rods }}$.

The assume that $M_{1}$-number of constraints of type

$$
u_{x i k}-u_{x j n}=0, k, n \in[1,2 \ldots N], i, j \in[1,2]
$$

are set on the system, which Express the conditions of equal displacement $u_{x i k}$ and $u_{x j n}$. Let us add $M_{2}$-number of constraints to primary set to represent displacement boundary conditions

$$
u_{\alpha i k}=u_{\alpha i k}^{*}(t), \theta_{\alpha i k}=\theta_{\alpha i k}^{*}(t), k \in[1,2 . . N], i \in[1,2], \alpha=x, y, z
$$

here $u_{\alpha i k}^{*}(t), \theta_{\alpha i k}^{*}(t)$ - prescribed displacements and rotation angles of the sections.

Due to linearity of relations (4), (5), they can be represented as the matrix type:

$$
\mathrm{CU}=\mathbf{U}^{*}(t)
$$

here matrix relating nodal dof to structure dof (matrix links) $\mathrm{C}$ has a size of $\left(M_{2}+\right.$ 
$\left.M_{2}\right) \times\left(12 \cdot N_{\text {Rods }}\right)$.This matrix is in General rectangular and rarefied: it has two element in the line (1 и -1) - connection type (5) and one element in the string (1) - connection type (6).

We introduce the functional of the fine:

$$
S=\lambda^{T}\left(\mathrm{C} \mathbf{U}-\mathbf{U}^{*}\right)
$$

where $\boldsymbol{\lambda}$ - Lagrange multipliers, all - greater than zero.

We introduce the functional $\mathrm{F}$ for which conditions of minimum are the equations (3):

$$
F=\mathbf{U}^{T}(\mathrm{~K} \mathbf{U}-\mathbf{R})
$$

The functional $\mathrm{F}$ is the sum of works of external and internal forces to nodal displacements; the functional $S$ a can be interpreted as the finefor the violation of boundary conditions and conditions of jointness of cores in the design.

Here require the minimum sum $F+S$ :

$$
\begin{aligned}
& \frac{\partial(F+S)}{\partial \mathbf{U}}=\mathrm{K} \mathbf{U}-\mathbf{R}+\mathrm{C}^{T} \boldsymbol{\lambda}=0 \\
& \frac{\partial(F+S)}{\partial \boldsymbol{\lambda}}=\mathrm{C} \mathbf{U}-\mathbf{U}^{*}=0
\end{aligned}
$$

In order to define node displacements a system of linear equations with square size matrix and inhomogeneous right side is obtained:

$$
\begin{aligned}
& \mathbf{K}_{\lambda} \mathbf{U}_{\lambda}=\mathbf{R}_{\lambda}, \\
& \mathbf{K}_{\lambda}=\left[\begin{array}{cc}
\mathbf{K}_{c b} & \mathbf{C}^{T} \\
\mathbf{C} & 0
\end{array}\right] ; \mathbf{U}_{\lambda}=\left\{\begin{array}{l}
\mathbf{U} \\
\lambda
\end{array}\right\} ; \mathbf{R}_{\lambda}=\left\{\begin{array}{c}
\mathbf{R} \\
\mathbf{U}^{*}
\end{array}\right\}
\end{aligned}
$$

This algorithm makes it easy to model systems under variable structure with beyond design impacts such as changes in the structure: the destruction of individual links. [9, 10] For this you need to change the structure of the matrix links $\mathrm{C}$, excluding the destroyed connection. When are appearance of defects (cracks) in a separate rod, it is sufficient to change one stiffness matrix in quasidiagonality the matrix $\mathrm{K}$ corresponding to this rod. Of course, to obtain the decision values (nodal displacements) is required after each change of the structure of the rod system.

Thus, to solve one problem (for a given structure of the system of rods), you need to build expressions for the nodal forces and the nodal displacements and set the matrix $\mathrm{C}$ that defines the system structure and kinematic boundary conditions

\section{The principle «One rod - one super element»}

As shown in Ch.1 the required condition of making FEM model of the rod system is an availability of expression allowing the estimation of node forces through node displacements. Vector of initial conditions consist of kinematic and force part:

$$
\begin{aligned}
& \mathbf{y}_{0}=\left\{\begin{array}{llllll}
\mathbf{y}_{0 C} & \mathbf{y}_{0 F}
\end{array}\right\} \\
& \mathbf{y}_{0 C}=\left\{\begin{array}{lllllll}
u_{0} & v_{0} & w_{0} & \theta_{x 0} & \theta_{y 0} & \theta_{z 0}
\end{array}\right\} \\
& \mathbf{y}_{0 F}=\left\{\begin{array}{lllllll}
M_{x 0} & M_{y 0} & M_{z 0} & N_{0} & Q_{y 0} & Q_{z 0}
\end{array}\right\}
\end{aligned}
$$

Then the expression for the static condition can also be represented in the form of a parts: 


$$
\begin{gathered}
\mathbf{y}_{C}(x)=\mathrm{V}_{C C}^{s t}(x) \mathbf{y}_{0 C}+\mathrm{V}_{C F}^{s t}(x) \mathbf{y}_{0 F}-\mathbf{F}_{q C}(x) \\
\mathbf{y}_{F}(x)=\mathrm{V}_{F C}^{s t}(x) \mathbf{y}_{0 C}+\mathrm{V}_{F F}^{s t}(x) \mathbf{y}_{0 F}-\mathbf{F}_{q F}(x) \\
\mathrm{V}_{C C}^{s t}(x)=\left[\mathrm{V}^{s t}\right]_{1.6,1 . .6} ; \mathrm{V}_{C F}^{s t}(x)=\left[\mathrm{V}^{s t}\right]_{1.6,7 . .12} \\
\mathrm{~V}_{F C}^{s t}(x)=\left[\mathrm{V}^{s t}\right]_{7 . .12,1 . .6} ; \mathrm{V}_{F F}^{s t}(x)=\left[\mathrm{V}^{s t}\right]_{7 . .12,7 . .12}
\end{gathered}
$$

Eliminate the force factors at the beginning of the rod through the displacement of its end, recording the first equality of (12) to the end of the rod in case $x=L$ :

$$
\begin{gathered}
\mathbf{y}_{C}(L)=\mathbf{y}_{k C}=\mathrm{V}_{C C}^{s t}(L) \mathbf{y}_{0 C}+\mathrm{V}_{C F}^{s t}(L) \mathbf{y}_{0 F}-\mathbf{F}_{q C}(L) \Rightarrow \\
\Rightarrow \mathbf{y}_{0 F}=\mathrm{V}_{C F}^{s t}(L)^{-1}\left\{\mathbf{y}_{k C}-\mathrm{V}_{C C}^{s t}(L) \mathbf{y}_{0 C}+\mathbf{F}_{q C}(L)\right\}
\end{gathered}
$$

Now substitute the result in (12) and obtain the expression state at any point of the rod using the nodal displacements:

$$
\begin{aligned}
& \mathbf{y}_{C}(x)=\mathrm{V}_{C C}^{s t}(x) \mathbf{y}_{0 C}+ \\
& +\mathrm{V}_{C F}^{s t}(x) \mathrm{V}_{C F}^{s t}(L)^{-1}\left\{\mathbf{y}_{k C}-\mathrm{V}_{C C}^{s t}(L) \mathbf{y}_{0 C}+\mathbf{F}_{q C}(L)\right\}-\mathbf{F}_{q C}(x)= \\
& =\left\{\mathrm{V}_{C C}^{s t}(x)-\mathrm{V}_{C F}^{s t}(x) \mathrm{V}_{C F}^{s t}(L)^{-1} \mathrm{~V}_{C C}^{s t}(L) \mathbf{y}_{0 C}+\mathrm{V}_{C F}^{s t}(x) \mathrm{V}_{C F}^{s t}(L)^{-1} \mathbf{y}_{k C}-\right. \\
& -\mathbf{F}_{q C}(x)+\mathrm{V}_{C F}^{s t}(x) \mathrm{V}_{C F}^{s t}(L)^{-1} \mathbf{F}_{q C}(L) \\
& \mathbf{y}_{F}(x)=\mathrm{V}_{F C}^{s t}(x) \mathbf{y}_{0 C}+ \\
& +\mathrm{V}_{F F}^{s t}(x) \mathrm{V}_{C F}^{s t}(L)^{-1}\left\{\mathbf{y}_{k C}-\mathrm{V}_{C C}^{s t}(L) \mathbf{y}_{0 C}+\mathbf{F}_{q C}(L)\right\}-\mathbf{F}_{q F}(x)= \\
& =\left\{\mathrm{V}_{F C}^{s t}(x)-\mathrm{V}_{F F}^{s t}(x) \mathrm{V}_{C F}^{s t}(L)^{-1} \mathrm{~V}_{C C}^{s t}(L) \mathbf{y}_{0 C}+\mathrm{V}_{F F}^{s t}(x) \mathrm{V}_{C F}^{s t}(L)^{-1} \mathbf{y}_{k C}-\right. \\
& -\mathbf{F}_{q F}(x)+\mathrm{V}_{F F}^{s t}(x) \mathrm{V}_{C F}^{s t}(L)^{-1} \mathbf{F}_{q C}(L)
\end{aligned}
$$

Let us write the expression (15) for the start $(x=0)$ and the end $(x=L)$ rod:

$$
\begin{aligned}
& \mathbf{y}_{F}(0)=\left\{\mathrm{V}_{F C}^{s t}(0)-\mathrm{V}_{F F}^{s t}(0) \mathrm{V}_{C F}^{s t}(L)^{-1} \mathrm{~V}_{C C}^{s t}(L)\right\} \mathbf{y}_{0 C}+\mathrm{V}_{F F}^{s t}(0) \mathrm{V}_{C F}^{s t}(L)^{-1} \mathbf{y}_{k C}- \\
& -\mathbf{F}_{q F}(0)+\mathrm{V}_{F F}^{s t}(0) \mathrm{V}_{C F}^{s t}(L)^{-1} \mathbf{F}_{q C}(L) ; \\
& \mathbf{y}_{F}(L)=\left\{\mathrm{V}_{F C}^{s t}(L)-\mathrm{V}_{F F}^{s t}(L) \mathrm{V}_{C F}^{s t}(L)^{-1} \mathrm{~V}_{C C}^{s t}(L)\right\}_{0 C}+\mathrm{V}_{F F}^{s t}(L) \mathrm{V}_{C F}^{s t}(L)^{-1} \mathbf{y}_{k C}- \\
& -\mathbf{F}_{q F}(L)+\mathrm{V}_{F F}^{s t}(L) \mathrm{V}_{C F}^{s t}(L)^{-1} \mathbf{F}_{q C}(L)
\end{aligned}
$$

In the left part (16) are the nodal moments and forces. Combine them into the vector of nodal power factors:

$$
\mathbf{F}_{\text {node }}=\left\{\mathbf{y}_{F}(0) \quad \mathbf{y}_{F}(L)\right\}
$$

Similarly dealing with the movements of the ends of the rod, combining them into a vector of nodal displacements:

$$
\mathbf{U}_{\text {node }}=\left\{\mathbf{y}_{0 C}(0) \quad \mathbf{y}_{k C}\right\}
$$

Then (16) can be written compactly:

$$
\mathbf{F}_{\text {node }}=\mathrm{KU}_{\text {node }}-\mathbf{F}_{q}^{\text {node }}
$$

Here are introduced the stiffness matrix of the rod: 


$$
\mathrm{K}=\left[\begin{array}{cc}
\mathrm{V}_{F C}^{s t}(0)-\mathrm{V}_{F F}^{s t}(0) \mathrm{V}_{C F}^{s t}(L)^{-1} \mathrm{~V}_{C C}^{s t}(L) & \mathrm{V}_{F F}^{s t}(0) \mathrm{V}_{C F}^{s t}(L)^{-1} \\
\mathrm{~V}_{F C}^{s t}(L)-\mathrm{V}_{F F}^{s t}(L) \mathrm{V}_{C F}^{s t}(L)^{-1} \mathrm{~V}_{C C}^{s t}(L) & \mathrm{V}_{F F}^{s t}(L) \mathrm{V}_{C F}^{s t}(L)^{-1}
\end{array}\right]
$$

and the vector of nodal loads from the external loads on the rod:

$$
\mathbf{F}_{q}^{\text {node }}=\left\{\mathbf{F}_{q F}(0)+\mathrm{V}_{F F}^{s t}(0) \mathrm{V}_{C F}^{s t}(L)^{-1} \mathbf{F}_{q C}(L) \quad \mathbf{F}_{q F}(L)+\mathrm{V}_{F F}^{s t}(L) \mathrm{V}_{C F}^{s t}(L)^{-1} \mathbf{F}_{q C}(L)\right\}
$$

The expression (19) the form is the same as analogous equation of the FEM and, therefore, it is possible to apply a known algorithm of the FEM in form the displacement method.

Note that for the spectral problem you can do the same calculations as above and get the result the same as in (19), with the exception of equality to zero nodal forces $\mathbf{F}_{q}^{\text {node }}$ :

$$
\mathbf{F}_{\text {node }}\left(\omega_{n}\right)=\mathrm{K}\left(\omega_{n}\right) \mathbf{Y}_{\text {node }}\left(\omega_{n}\right)
$$

here $\mathbf{Y}_{\text {node }}$ - vector of nodal displacements corresponding to the natural frequency $\omega \mathrm{n}$.

The result obtained is FE or SE? [11] Apparently, it is the super-element of a straight rod, as its original ratio do not contain internal degrees of freedom. He models an element of a subsystem - one rod with its structural features. To interpret the results compute nodal displacements should be used (14), (15), rewriting them in matrix form:

$$
\mathbf{y}(x)=\mathrm{D}(x) \mathbf{U}^{\text {node }}-\mathbf{F}(x)
$$

where

$$
\begin{aligned}
& \mathrm{D}(x)=\left[\begin{array}{ll}
\mathrm{V}_{C C}^{s t}(x)-\mathrm{V}_{C F}^{s t}(x) \mathrm{V}_{C F}^{s t}(L)^{-1} \mathrm{~V}_{C C}^{s t}(L) & \mathrm{V}_{C F}^{s t}(x) \mathrm{V}_{C F}^{s t}(L)^{-1} \\
\mathrm{~V}_{F C}^{s t}(x)-\mathrm{V}_{F F}^{s t}(x) \mathrm{V}_{C F}^{s t}(L)^{-1} \mathrm{~V}_{C C}^{s t}(L) & \mathrm{V}_{F F}^{s t}(x) \mathrm{V}_{C F}^{s t}(L)^{-1}
\end{array}\right] \\
& \mathbf{F}(x)=\left\{\begin{array}{l}
\mathbf{F}_{q C}(x)-\mathrm{V}_{C F}^{s t}(x) \mathrm{V}_{C F}^{s t}(L)^{-1} \mathbf{F}_{q C}(L) \\
\mathbf{F}_{q F}(x)-\mathrm{V}_{F F}^{s t}(x) \mathrm{V}_{C F}^{s t}(L)^{-1} \mathbf{F}_{q C}(L)
\end{array}\right\}
\end{aligned}
$$

\section{Conclusion}

Similarly, should seek own of the condition.

The interpretation of the results is the most time-consuming part of the computational work due to the necessity of calculating the influence matrices to determine the weak sections of the rod. At the same time, for typical structural elements possible to perform cataloging stiffness matrix $\mathrm{K}$ and interpretation matrix $\mathrm{D}$ for use in calculating of other structures, possessing the same elements. [12]

Solution of inhomogeneous dynamic problems for the entire load-bearing structure should be carried out after determining its spectrum.

\section{References}

1. O.A. Kovalchuk, Natural and technical Sciences, 11-12, 344-348 (2014)

2. R. W. Clough, Computers and Structures, 12, 361-370 (1980)

3. E.I. Sorokina, K.M. Melikhov, L.N. Makovkina, Technical science in Russia and abroad, 4, 134-137 (2015)

4. E.E. Krasnovsky, A.S. Shadskiy, Bulletin of Moscow state technical University N. Uh. Bauman, S, 169-176 (2011) 
5. Kai-Liang Lu, Yuan Liu, Wei-guo Zhang, Hui-qing Qiu, Wei-jian Mi, Procedia Engineering, 16, 685-694 (2011)

6. O.A. Kovalchuk, Building: Science and Education, 1, 1-6 (2012)

7. S.S. Law, T.H.T. Chan, D. Wu, Journal of Sound and Vibration, 239-1, 19-39 (2001)

8. A.G. Tamrazyan, O.A. Kovalchuk, National Research Center Bulletin, 3-4, 120-130 (2011)

9. V.M. Bondarenko, V.I. Kolchunov, E.D. Vorobyov, The Bulletin of the construction technology, 1, 8-11, (2004)

10. V.D. Rayzer, Construction mechanics and calculation of structures, 5, 77-78, (2012)

11. O.A. Kovalchuk, Industrial and Civil Engineering, 8, 70-73 (2016)

12. I. Cismasiu, J.P. Moitinho de Almeida, Advances in Engineering Software, 38, 11-12, 750-762 (2007) 